\title{
1 The Ensembl COVID-19 resource: Ongoing integration of public 2 SARS-CoV-2 data
}

3

4

Nishadi H. De Silva, Jyothish Bhai, Marc Chakiachvili, Bruno Contreras-Moreira, Carla Cummins, Adam Frankish, Astrid Gall, Thiago Genez, Kevin L. Howe, Sarah E. Hunt, Fergal J. Martin, Benjamin Moore, Denye Ogeh, Anne Parker, Andrew Parton, Magali Ruffier, Manoj Pandian Sakthivel, Dan Sheppard, John Tate, Anja Thormann, David Thybert, Stephen J. Trevanion, Andrea Winterbottom, Daniel R. Zerbino, Robert D. Finn, Paul Flicek, Andrew D. Yates*

European Molecular Biology Laboratory, European Bioinformatics Institute, Wellcome Genome Campus, Hinxton, Cambridge CB10 1SD, United Kingdom

*To whom correspondence should be addressed. Tel: +44(0)1223492538 Email: ayates@ebi.ac.uk

\section{ABSTRACT}

The COVID-19 pandemic has seen unprecedented use of SARS-CoV-2 genome sequencing for epidemiological tracking and identification of emerging variants. Understanding the potential impact of these variants on the infectivity of the virus and the efficacy of emerging therapeutics and vaccines has become a cornerstone of the fight against the disease. To support the maximal use of genomic information for SARS-CoV-2 research, we launched the EnsembI COVID-19 browser, incorporating a new Ensembl gene set, multiple variant sets (including novel variation calls), and annotation from several relevant resources integrated into the reference SARS-CoV-2 assembly. This work included key adaptations of existing Ensembl genome annotation methods to model ribosomal slippage, stringent filters to elucidate the highest confidence variants and utilisation of our comparative genomics pipelines on viruses for the first time. Since May 2020, the content has been regularly updated and tools such as the Ensembl Variant Effect Predictor have been integrated. The Ensembl COVID-19 browser is freely available at https://covid-19.ensembl.org.

\section{INTRODUCTION}

Over the past twenty years, multiple zoonotic respiratory diseases caused by coronaviruses have been identified. Examples include the SARS epidemic caused by severe acute respiratory syndrome coronavirus (SARS-CoV) in 2003 and the Middle East respiratory syndrome coronavirus (MERS-CoV) outbreak in 2012. Both belong to the betacoronavirus genus and are believed to have originated in bats with an intermediary animal host before transmission to humans ${ }^{1}$. 
The Ensembl COVID-19 resource: Ongoing integration of public SARS-CoV-2 data

36 The SARS-CoV-2 virus responsible for the current COVID-19 pandemic is also a betacoronavirus,

37 with a 29,903-nucleotide positive-strand RNA genome encoding $\sim 30$ known and hypothetical mature

38 proteins. The first open reading frame (ORF), representing approximately $67 \%$ of the entire genome,

39 encodes 16 non-structural proteins (nsps). The remaining ORFs encode accessory proteins and four

40 major structural proteins: spike surface glycoprotein (S), small envelope protein (E), matrix protein (M)

41 and nucleocapsid protein $(\mathrm{N})$.

43 Genomic sequencing has played a crucial role in understanding the mechanisms, spread and

44 evolution of this virus. In the UK alone, at the time of writing, close to $5 \%$ of all reported infections

45 each week were being sequenced (COG-UK, January 2021: https://www.cogconsortium.uk/wp-

46 content/uploads/2021/02/COG-UK-geo-coverage_2021-02-01_summary.pdf) and this trend is likely to

47 grow. Established genomic resources, such as Ensembl, have been able to leverage these data and

48 bring them to new and existing user communities supporting research leveraging the rapidly emerging

49 SARS-CoV-2 data landscape.

51 Ensemb| $\left.\right|^{2,3}$ was launched to capture data from the Human Genome Project and has since developed 52 into a large scale system for generating, integrating and disseminating genomic information. The

53 COVID-19 pandemic presented new challenges related to presenting SARS-CoV-2 annotation and

54 data within Ensembl. Meeting these, we launched the Ensembl COVID-19 browser (https://covid-

55 19.ensembl.org) in May 2020 using concepts and workflows that enable rapid update cycles to react 56 quickly in the face of new data and potential future outbreaks.

\section{NEW ENSEMBL COVID RESOURCE}

\section{Reference assembly and a new gene annotation}

61 The SARS-CoV-2 sequence represented in Ensembl (INSDC accession GCA_009858895.3,

62 MN908947.3) is the viral RNA genome isolated from one of the first cases in Wuhan, China ${ }^{4}$. It is

63 widely used as the standard reference and has been incorporated into other resources such as the

64 UCSC SARS-CoV-2 genome browser ${ }^{5}$. This assembly was imported from the European Nucleotide 
The Ensembl COVID-19 resource: Ongoing integration of public SARS-CoV-2 data

65 Archive (ENA) into an Ensembl database schema with minor modifications to software regularly used

66 to integrate assemblies from the ENA into Ensembl.

67

68 To enable the correct annotation of SARS-CoV-2, the Ensembl gene annotation methods ${ }^{6}$ were

69 adapted to reflect the biology of the virus. To identify protein coding genes, we aligned SARS-CoV-2

70 proteins from RefSeq ${ }^{7}$ to the genome using Exonerate ${ }^{8}$. A challenge for annotation is that the first and

71 largest ORF can result in either non-structural proteins nsp1-11 (ORF1a) or in nsp1-nsp10 and

72 nsp12-nsp16 (ORF1ab) via an internal programmed translational frameshift ${ }^{9}$. Exonerate handles this

73 ribosomal slippage by inserting a gap in the alignment and thus allowing the annotation of the full

74 ORF1ab locus. Our modified annotation methodology then removes the artificial gap to represent the

75 slippage frameshift as an RNA edit and ensures a biologically accurate representation of the locus

76 and product.

78 Our annotation approach was tested on 90 additional SARS-CoV-2 assemblies retrieved from the

79 ENA. We assessed alignment coverage and percentage identity of the resultant gene translations to

80 verify accuracy and consistency. In all cases, full length alignments were observed and average

81 amino acid percentage identity across all genes in most assemblies were $99.9 \%$ or $100 \%$ (one

82 assembly had $99.81 \%$ identity). These results demonstrate that our annotation approach is able to

83 scale consistently to larger volumes of viral data.

85 In addition to generating a fully integrated Ensembl gene annotation, we also imported the gene set

86 submitted to the ENA with the reference sequence by the Shanghai Public Health Clinical Centre. As

87 shown in figure 1, both the submitted (blue) and the Ensembl gene annotations (red) can be viewed

88 simultaneously on the browser. The submitted gene annotation is displayed as a separate annotation

89 track, accessed under the 'Genes and transcripts' heading after clicking on 'Configure this page' in

90 the left-hand menu. The major difference between the annotations is that the submitted annotation

91 does not include the short form ORF1a or ORF7b. 
bioRxiv preprint doi: https://doi org/10.1101/2020.12.18.422865 this version posted March 29 2021. The copyright holder for this preprint (which was not certified by peer review) is the author/funder, who has granted bioRxiv a license to display the preprint in perpetuity. It is made available under aCC-BY 4.0 International license.

The Ensembl COVID-19 resource: Ongoing integration of public SARS-CoV-2 data

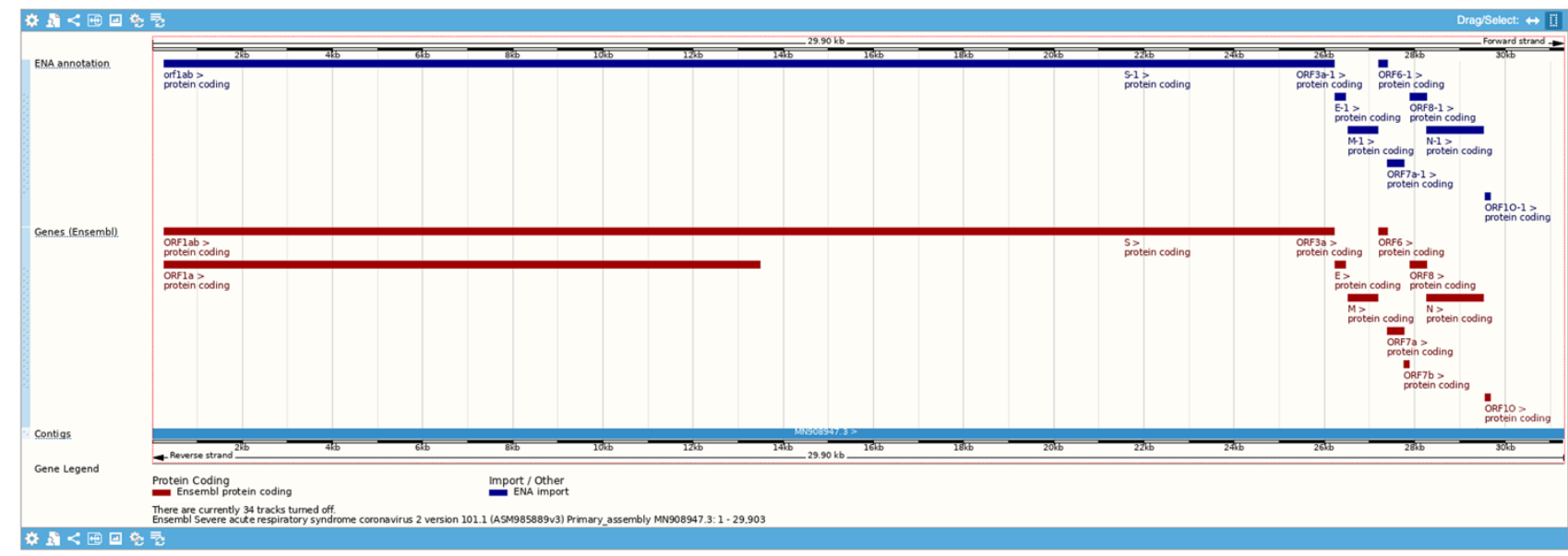

Figure 1: A comparison of the Ensembl gene set and the gene set submitted to the ENA by the Shanghai Public Health Clinical Centre for the SARS-CoV-2 reference assembly

\section{Comparison of SARS-CoV-2 with 60 other Orthocoronavirinae genomes}

100

We used Cactus ${ }^{11}$ to align SARS-CoV-2 to 60 publicly available virus genomes from the

102 Orthocoronavirinae subfamily. The results showed $78 \%$ of the SARS-CoV-2 genome aligned with at

103 least one other genome and $35 \%$ of the genome aligned with the complete set of Orthocoronavirinae

104 genomes. The multiple sequence alignment gives evolutionary context for each region of the genome

105 and is a powerful method to explore functionality. For example, comparative genomics information

106 such as this can be used for analyses such as a recent comparison of the gene sets of 44 complete

107 Sarbecovirus genomes suggesting both a potentially novel alternate frame gene ORF3c and that

108 ORF10, ORF9c, ORF3b and ORF3d are unlikely to be protein coding ${ }^{10}$.

110 The alignment coverage (see figure 2) represents the number of genomes aligned to a given

111 reference genomic position and is distributed heterogeneously across the SARS-CoV-2 genome. An

112 immediate observation is that the central region of the genome (starting from $\sim 7.1 \mathrm{~kb}$ and ending at

$11321.3 \mathrm{~kb}$ ), including a significant segment of the 3' part of ORF1a, is highly shared across the

114 Orthocoronavirinae subfamily. This indicates that the non-structural proteins encoded by this region

115 (nsp3 - nsp16) likely originate from the Orthocoronavirinae ancestral genome. Conversely, both ends

116 of the SARS-CoV-2 genome have very low alignment coverage and are only shared with closely

117 related viruses. As a further demonstration of the utility of the alignment coverage, we focused in on

118 the genomic region encoding for the SARS-CoV-2 spike protein (figure 2). The spike protein has two

119 subunits: S1 which binds to the host cell receptor angiotensin-converting enzyme 2 (ACE2) and S2, 
bioRxiv preprint doi: https://doi org/10.1101/2020.12.18.422865; this version posted March 29, 2021. The copyright holder for this preprint (which was not certified by peer review) is the author/funder, who has granted bioRxiv a license to display the preprint in perpetuity. It is made available under aCC-BY 4.0 International license.

The Ensembl COVID-19 resource: Ongoing integration of public SARS-CoV-2 data

120 which is involved in membrane fusion. The region of the S ORF encoding for the S2 subunit of the

121 spike protein clearly displays a high alignment coverage while the region encoding for the S1 subunit

122 has large portions that are shared only by one other related genome. This demonstrates the dramatic

123 difference in conservations between the S1 and S2 subunits.

124

125

126

127

128

129

130

131

132

133

134

135

136

\section{Genetic variation data}

138 Analysis of genetic variants of viral genomes is important for understanding the spread of infection

One set comes from the Nextstrain project which creates phylogenetic trees for tracking pathogen

Figure 2: Alignment coverage across the SARS-CoV-2 reference genome based on a multiple sequence alignment with 60 other Orthocoronavirinae genomes. The green plot of alignment coverage shows that the central region of the genome is highly shared across the subfamily, while the ends are generally shared only with closely related viruses. The region encoding for the spike protein $\mathrm{S}$ has been highlighted within the red circle showing the difference between the low alignment coverage of the upstream S1 subunit and the high coverage of the downstream S2 subunit.

Additionally, we applied our gene tree method ${ }^{12}$ to group the protein coding genes into families and to predict orthologous and paralogous relationships between genes. These results will be incorporated into the COVID-19 resource in Q2 2021. across different geographic regions. We display 6,134 sequence variants for SARS-CoV-2 and show their regional frequency distributions alongside predicted molecular consequences calculated by the Ensembl Variant Effect Predictor (VEP) ${ }^{13}$. The variants on our site are derived from overlapping sample sets produced by two groups and a small collection of variants of special interest. evolution based on virus subsamples ${ }^{14}$. We converted their SARS-CoV-2 data release from 08-04- 
bioRxiv preprint doi: https://doi org/10.1101/2020.12.18.422865; this version posted March 29, 2021. The copyright holder for this preprint (which was not certified by peer review) is the author/funder, who has granted bioRxiv a license to display the preprint in perpetuity. It is made available under aCC-BY 4.0 International license.

The Ensembl COVID-19 resource: Ongoing integration of public SARS-CoV-2 data

1462020 to VCF for integration into our system and display frequency distributions by country and

147 Nextstrain-inferred clade.

148

149 The second variant set comes from the ENA team, who developed a LoFreq-based ${ }^{15}$ pipeline to call

150 variants from SARS-CoV-2 sequence data sets submitted to their archives. LoFreq reports the

151 proportion of each variant seen in a sample from an individual. For simplicity, we represent only the

152 alleles seen in each sample and not the proportions estimated. Variants were called for each host

153 sample individually and, to provide a more accurate estimation of the frequency of each allele across

154 the entire sample set, it is assumed that sites at which a variant was not called in a sample match the

155 reference genome used in the Ensembl COVID-19 browser. We currently display ENA's variant data

156 from 17-08-2020 and have applied strict filters to reduce the proportion of lower confidence sites.

157 Specifically, we have not included variants from sequence data sets with more than 40 calls and we

158 have removed variants where no sample has a frequency of $20 \%$ or more for the non-reference allele

159 and variants where all samples show strand bias.

160

161 Some sites are annotated as a further guide to quality. For example, variants seen in more than one

162 sample in either set have an evidence status of 'Multiple observations' and variants at sites

163 recommended for masking by De Maio et $a{ }^{16}$ have a flag of 'Suspect reference location'. Variants can

164 be displayed as three separate tracks in the genome browser: those from ENA, those from Nextstrain

165 and those observed in more than one sample in either project as shown in figure 3.

167 We also display a set of variants which were reported as a tracking priority by the COVID-19

168 Genomics UK Consortium (COG-UK, https://www.cogconsortium.uk/) in December 2020. This

169 includes 17 variants from the rapidly spreading B.1.1.7 strain (https://virological.org/t/preliminary-

170 genomic-characterisation-of-an-emergent-sars-cov-2-lineage-in-the-uk-defined-by-a-novel-set-of-

171 spike-mutations/563) and four variants from the mink associated strain. The D614G, A222V and

172 N439K mutations associated with an effect on transmissibility, a fast growing lineage and increased

173 binding affinity to the ACE2 receptor ${ }^{17-19}$, respectively, have also been included. We extracted the

174 gene and protein change information from the reports and used the Ensembl VEP to map these

175 descriptions to genomic coordinates and create a VCF file, which was then loaded into the Ensembl 
The Ensembl COVID-19 resource: Ongoing integration of public SARS-CoV-2 data

176 database with associated phenotype information. These variants can be viewed as a 'COG-UK

177 priority mutations' track alongside the gene annotations.

\section{Integration of data from other resources}

179 To enrich the SARS-CoV-2 genome annotation we aligned and integrated data from several external

180 repositories in a similar manner to other genomes available in Ensembl.

181 Specifically, we aligned Rfam $^{20}$ covariance models using their COVID-19 release 14.2

182 (http://rfam.xfam.org/covid-19) to highlight conserved non-coding RNA structures which are

183 responsible for various stages of the viral life cycle. These include the frame shifting stimulation element and the pseudoknot necessary for the genome replication of SARS-CoV- ${ }^{21}$. We also provide cross references to proteins from RefSeq, UniProt ${ }^{22}$ and the International Nucleotide Sequence Database Collaboration (INSDC); functional annotation from the Gene Ontology Consortium; and

187 annotation of protein domains using InterProScan. These additional annotations are accessible via our region views and the gene and transcript tabs. We also created a genome browser track projecting the protein-domain annotations onto the genome to facilitate a genome-oriented view of the gene products including the non-structural cleavage products of ORF1a/ORF1ab.

192 The browser also displays community annotation of sites and regions using results co-ordinated by

193 the UCSC genome browser. Additions to this annotation resource are open to all and done via a

194 publicly available spreadsheet hosted by UCSC (http://bit.ly/cov2annots), the data from which is 195 integrated periodically into the Ensembl browser. This is achieved via specialised code that uses Git 196 workflows to convert the annotations into BigBed files that can be visualised on a variety of genome 197 browsers (available freely at https://github.com/Ensembl/sarscov2-annotation).

198 We have integrated Oxford Nanopore sequencing primers (version 3) made available by the ARTIC 199 network (https://artic.network/ncov-2019) to assist in sequencing the virus. Though mainly focused on 200 the Oxford Nanopore MinION sequencer, some aspects of the protocol may be generalised to other 201 sequencing platforms. The complete list of primers included is available on GitHub 202 (https://github.com/artic-network/artic-ncov2019/blob/master/primer_schemes/nCoV-2019/V3/nCoV- 
bioRxiv preprint doi: https://doi.org/10.1101/2020 12.18.422865; this version posted March 29, 2021. The copyright holder for this preprint (which was not certified by peer review) is the author/funder, who has granted bioRxiv a license to display the preprint in perpetuity. It is made available under aCC-BY 4.0 International license.

The EnsembI COVID-19 resource: Ongoing integration of public SARS-CoV-2 data

204 Finally, we provide tracks to visualise problematic and caution sites, which result from common 205 systematic errors associated with laboratory protocols and have been observed in submitted 206 sequences ${ }^{16}$. Inclusion of these can adversely influence phylogenetic and evolutionary inference. 207 Visualising these in the browser alongside the locations of primers and other community derived 208 annotations helps determine how best to proceed with analyses of each these sites. altered the vocabulary wherever possible and are reviewing feedback as we receive it.

Figure 3: The browser with several tracks turned on and highlighting a substitution flagged up early on in the UCSC community annotation at position 23403 (D614G) in the S spike glycoprotein gene. Due to the prompt nature of community driven annotation, this data was available on our browser as soon as the annotation appeared in a preprint. It is labelled as a common missense mutation in SARS-CoV-2 with a notably high difference in resulting isoelectric point (D->G). Pachetti et al (2020) looked at 220 genomic sequences obtained from the GISAID database and characterised 8 novel recurrent mutations; the one at 23403 is one of them. Many studies now show that this particular missense mutation in the spike protein is predominantly observed in Europe ${ }^{23}$; patterns that can also be seen in the variation data we host.

\section{Integration and engagement}

The Ensembl COVID-19 resource features a newly designed landing page, which prioritises key views and data to help direct researchers into relevant sections of the site. To support expeditious data release, we have not made potentially time-consuming virus-specific modifications to our existing web codebase — such as showing a single nucleic acid strand and removing all mentions of exonsbecause we felt the data could be effectively understood without these changes. However, we have

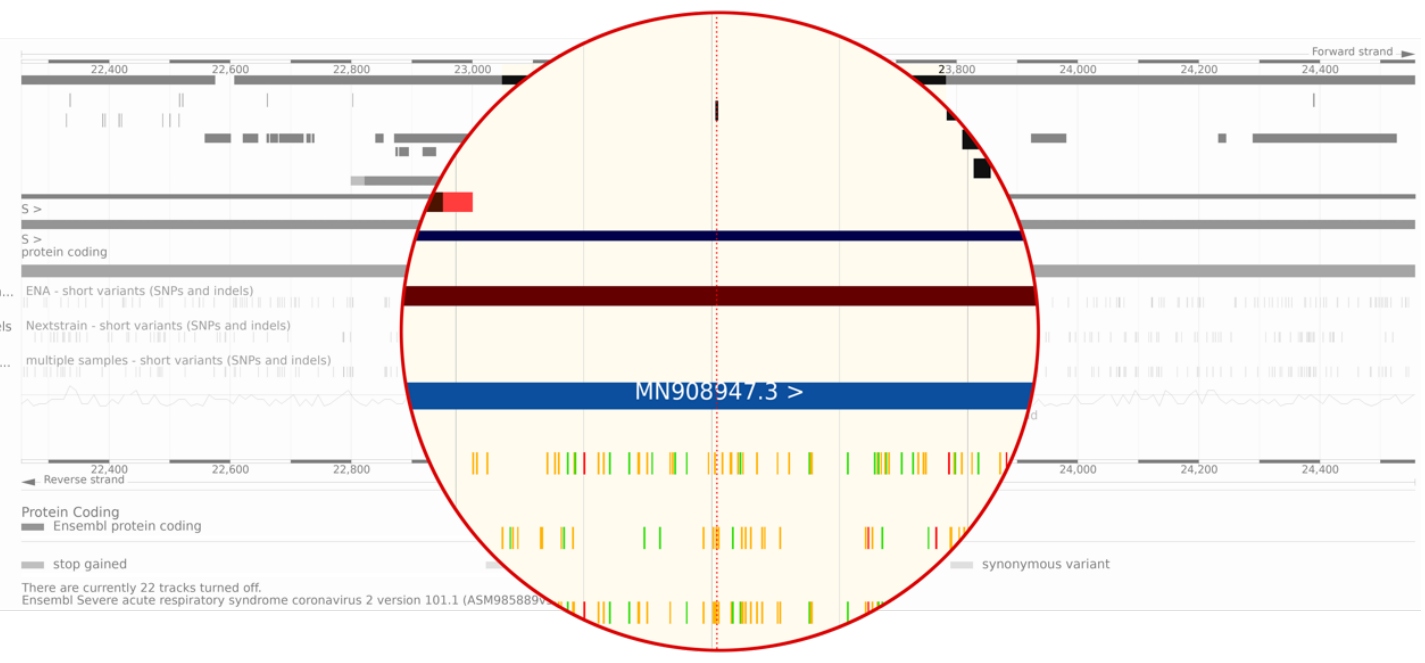


The Ensembl COVID-19 resource: Ongoing integration of public SARS-CoV-2 data

228 Our COVID-19 resource is also integrated into the European COVID-19 Data Portal hosted by EMBL-

229 EBI (https://www.covid19dataportal.org/). The portal enables searches across the multiple research

230 outputs on COVID-19 including viral and human sequences; relevant biochemical pathways,

231 interactions, complexes, targets and compounds; protein and expression data; and literature.

232

233 We have engaged our existing and new user communities using our blog and social media accounts

234 to announce the release and updates to the Ensembl COVID-19 resource. We also highlighted

235 the changes made to our gene annotation method to ensure the complete set of ORFs because these

236 have been overlooked by other annotation tools.

\section{DISCUSSION}

239 The swift spread of COVID-19 has highlighted the necessity for data resources to be prepared for

240 rapid adaptation to developing outbreaks. Our development and release of the Ensembl COVID-19

241 resource leveraged our experience integrating thousands of genomes into the Ensembl infrastructure

242 and supporting hundreds of thousands of users. The Ensembl COVID-19 browser provides a unique

243 view on SARS-CoV-2 using our gene annotation method and variation data processed to focus on the

244 highest confidence variants. Additionally, the Ensembl VEP and haplotype views enable the

245 consequences of the variants to be assessed within the context of specific strains and geographical

246 locations. The data is made accessible via the widely used Ensembl platform making it immediately

247 familiar to a large userbase who may be able to repurpose existing software and browser knowledge

248 to support their work during the pandemic and beyond.

250 When the COVID-19 pandemic hit, we had been working for several months to develop Ensembl

251 Rapid Release (https://rapid.ensembl.org) to distribute annotated genomes within days of their

252 annotation being completed. This experience proved useful in bringing the COVID-19 site to public

253 release quickly. We have also demonstrated the flexibility of the Ensembl infrastructure and its value

254 as a platform for research and discovery. Indeed, all of our pipelines and schemas worked seamlessly

255 even though Ensembl was not designed to support RNA genomes and had not previously been used

256 for viruses. The adapted gene annotation method, for instance, produced consistent annotation with

257 ribosomal slippage correctly modelled and can be reused in the future. Similarly, the gene tree and 
The EnsembI COVID-19 resource: Ongoing integration of public SARS-CoV-2 data

258

259

260

261

262

263

264

265

266

267

268

269

270

271

272

273

274

275

276

277

278

279

280

281

282

283

284

285

286

287

alignment pipelines have been applied to the viral data with only minimal changes to parameters. We will continue to regularly update the site as new data emerges to support research into understanding the genomic evolution of this virus, identifying hotspots of genomic variation and enabling the rational design of future therapeutics, vaccines and policies well beyond the end of the current pandemic.

\section{REFERENCES}

1. Wu, A. et al. Genome Composition and Divergence of the Novel Coronavirus (2019-nCoV) Originating in China. Cell Host Microbe 27, 325-328 (2020).

2. Howe, K. L. et al. Ensembl Genomes 2020—enabling non-vertebrate genomic research. Nucleic Acids Res. 48, D689-D695 (2020).

3. Howe, K. L. et al. Ensembl 2021. Nucleic Acids Res. 49, D884-D891 (2021).

4. Wu, F. et al. A new coronavirus associated with human respiratory disease in China. Nature 579, 265-269 (2020).

5. Fernandes, J. D. et al. The UCSC SARS-CoV-2 Genome Browser. Nat. Genet. 52, 991-998 (2020).

6. Aken, B. L. et al. The Ensembl gene annotation system. Database 2016, baw093 (2016).

7. O'Leary, N. A. et al. Reference sequence (RefSeq) database at NCBI: current status, taxonomic expansion, and functional annotation. Nucleic Acids Res. 44, D733-D745 (2016).

8. Slater, G. S. C. \& Birney, E. Automated generation of heuristics for biological sequence comparison. BMC Bioinformatics 6, (2005).

9. Chen, Y., Liu, Q. \& Guo, D. Emerging coronaviruses: Genome structure, replication, and pathogenesis. J. Med. Virol. 92, 418-423 (2020).

10. Jungreis, I., Sealfon, R. \& Kellis, M. SARS-CoV-2 gene content and COVID-19 mutation impact by comparing 44 Sarbecovirus genomes. bioRxiv (preprint) (2020) doi:10.1101/2020.06.02.130955.

11. Armstrong, J. et al. Progressive Cactus is a multiple-genome aligner for the thousand-genome era. Nature 587, 246-251 (2020).

12. Herrero, J. et al. Ensembl comparative genomics resources. Database 2016, bav096 (2016).

13. McLaren, W. et al. The Ensembl Variant Effect Predictor. Genome Biol. 17, 122 (2016). 
The EnsembI COVID-19 resource: Ongoing integration of public SARS-CoV-2 data

14. Hadfield, J. et al. Nextstrain: real-time tracking of pathogen evolution. Bioinformatics 34, 41214123 (2018).

15. Wilm, A. et al. LoFreq: a sequence-quality aware, ultra-sensitive variant caller for uncovering cell-population heterogeneity from high-throughput sequencing datasets. Nucleic Acids Res. 40, 11189-11201 (2012).

16. Nicola, D. M. et al. Issues with SARS-CoV-2 sequencing data. https://virological.org/t/issueswith-sars-cov-2-sequencing-data/473 (2020).

17. Volz, E. et al. Evaluating the Effects of SARS-CoV-2 Spike Mutation D614G on Transmissibility and Pathogenicity. Cell 184, 64-75.e11 (2021).

18. Bartolini, B. et al. The newly introduced SARS-CoV-2 variant A222V is rapidly spreading in Lazio region, Italy. medRxiv (preprint) doi:10.1101/2020.11.28.20237016.

19. Thomson, E. et al. The circulating SARS-CoV-2 spike variant N439K maintains fitness while evading antibody-mediated immunity. (2020) doi:10.1101/2020.11.04.355842.

20. Kalvari, I. et al. Rfam 14: expanded coverage of metagenomic, viral and microRNA families. Nucleic Acids Res. 49, D192-D200 (2021).

21. Williams, G. D., Chang, R.-Y. \& Brian, D. A. A Phylogenetically Conserved Hairpin-Type 3' Untranslated Region Pseudoknot Functions in Coronavirus RNA Replication. J. Virol. 73, 8349 LP - 8355 (1999).

22. Bateman, A. et al. UniProt: the universal protein knowledgebase in 2021. Nucleic Acids Res. 49, D480-D489 (2021).

23. Isabel, S. et al. Evolutionary and structural analyses of SARS-CoV-2 D614G spike protein mutation now documented worldwide. Sci. Rep. 10, 14031 (2020).

\section{ACKNOWLEDGEMENTS}

We would like to thank the following people at the EMBL-EBI for their contributions to our resource and thoughtful discussions: Nick Goldman, Zamin Iqbal, Guy Cochrane, Rodrigo Lopez Sanchez, Conor Walker, Nadim Rahman, Jeena Rajan, Alexy Sokolov, Peter Harrison, Youngmi Park, Nicola Buso, Suran Jayathilaka, Anton Petrov, James Allen, Luca Da Rin Fioretto, Thomas Maurel and Vinay Kaikala. 
The Ensembl COVID-19 resource: Ongoing integration of public SARS-CoV-2 data

318 This work was supported by the Wellcome Trust [WT108749/Z/15/Z] and the European Molecular

319 Biology Laboratory. For the purpose of open access, the authors have applied a CC BY public

320 copyright licence to any Author Accepted Manuscript version arising from this submission.

\section{AUTHOR CONTRIBUTIONS}

323 N.H.D.S., R.D.F., P.F., A.F., K.L.H., S.E.H., F.J.M., M.R., A.D.Y. and D.R.Z. conceptualised the

324 resource. N.H.D.S., S.E.H., F.J.M., M.R. and A.D.Y. contributed to the methodology. M.C., B.C., C.C.,

325 T.G., S.E.H., F.J.M., D.N.O., A Parker, A Parton, M.R., M.P.S., D.S., J.T. and A.T. developed the

326 software. M.C., C.C., N.H.D.S., A.G., T.G., M.R., D.T. and A.D.Y. validated the data while C.C., T.G.,

327 K.L.H., S.E.H., M.R. and D.T. conducted formal analysis on the computed results. C.C., T.G., D.N.O.

328 and D.T. helped with investigations of software and results. N.H.D.S. wrote the original draft of this

329 manuscript and R.D.F., P.F., A.F., A.G., K.L.H., S.E.H., B.M., A Parker, M.R., D.T., S.J.T., A.D.Y. and

330 D.R.Z. reviewed and edited it. A.W. created the visualisation for the resource landing page. N.H.D.S.,

331 R.D.F., P.F., K.L.H., S.E.H., M.R., S.J.T. and A.D.Y. supervised various aspects of the project. M.R.

332 and A.D.Y. were involved in project administration and K.L.H., P.F., A.D.Y. and D.R.Z. acquired funds

333 to support the project.

\section{COMPETING INTERESTS}

336 P.F. is a member of the scientific advisory boards of Fabric Genomics, Inc., and Eagle Genomics, Ltd.

DATA AVAILABILITY

339 The COVID-19 resource from Ensembl is available without restrictions at https://covid-

340 19.ensembl.org. The reference genome assembly for SARS-CoV-2 with the accession

341 GCA_009858895.3 was obtained from the European Nucleotide Archive

342 (https://www.ebi.ac.uk/ena/browser/view/GCA 009858895.3).

\section{CODE AVAILABILITY}

345 The selection of our code to convert CSV files into BigBed files is at

346 https://github.com/Ensembl/sarscov2-annotation. The code relevant to processing SARS-CoV-2

347 variants in Ensembl is at https://github.com/Ensembl/ensembl-variation, the gene annotation pipeline

348 is available at https://github.com/Ensembl/ensembl-annotation and the code used for comparative

349 analysis is at https://github.com/Ensembl/ensembl-compara 